\title{
Dexmedetomidine for Sedation and Analgesia in Mechanically Ventilated Patients
}

Md Nasir Uddin Ahmed ${ }^{1 *}$, Mayisha Afifa ${ }^{2}$, Mohammad Salim $^{3}$, Muhammad Asaduzzaman $^{4}$, Akm Ferdous Rahman $^{5}$

${ }^{1}$ Assistant Professor, Anwer Khan Modern Medical College, Dhaka, Bangladesh

${ }^{2}$ UN Physician, UN Clinic, Cox bazar, Bangladesh

${ }^{3,5}$ Assistant Professor, Dhaka Medical College, Dhaka, Bangladesh

${ }^{4}$ Assistant Professor, Kuwait Moitri Hospital, Dhaka, Bangladesh

DOI: $10.36347 /$ sjams.2021.v09i01.029

| Received: 09.01.2021 | Accepted: 20.01.2021 | Published: 29.01.2021

*Corresponding author: MD. Nasir Uddin Ahmed

Abstract

Original Research Article

Background: To reduce anxiety and pain, increase tolerance and improve outcomes of mechanically ventilated patients, sedation and analgesia are common practice. Over decades, $\gamma$-Aminobutyric acid (GABA) receptor agonist and opioids are used commonly for sedation and analgesia, respectively in intensive care unit (ICU). Preliminary evidence indicates that the $\alpha-2$ agonist dexmedetomidine may have distinct advantages to maintain both sedation and analgesia with minimal cardiovascular instability or respiratory depression, facilitate weaning from mechanical ventilation and decreases the duration of ICU stay. Aim of the study: The aim of this study was to assess the effectiveness of dexmedetomidine to the combined use of midazolam and fentanyl for sedation and analgesia in mechanically ventilated patients. Methods: This observational comparative study was conducted in the ICU of Dhaka Medical College Hospital (DMCH) at the Department of Anaesthesia, Analgesia \& Intensive Care Medicine during the period from March 2017 to June 2019. Total 146 mechanically ventilated patients were included in the study according to the selection criteria. Richmond agitation-sedation scale (RASS) and the Critical-care pain observation tool (CPOT) were used for assessment of sedation and analgesia respectively in this study. At first, the patients were divided into two groups and denoted as group A, and group B. In total 79 patients receiving Dexmedetomidine were denoted as in group A, and 67 patients receiving midazolam and fentanyl as combined were included into group B. Samples were randomization by fix card lottery method. All collected data were registered, documented and analyzed in the statistical program Statistical Package for Social Science (SPSS) version 22.0. Result: Among total 146 patients, the mean age of group A and group B were $45.56 \pm 13.35 \& 46.76 \pm 13.4$ (years), respectively. Both groups were similar in terms of age and gender distribution ( $p>0.05$ ). Dexmedetomidine was found more effective than midazolam and fentanyl combination for sedation. Percentage of times having desired RASS score were: group A 85.23 \pm 7.54 vs. group B 79.19 \pm 7.84 ; $(\mathrm{P}<0.05)$. While it was not much effective to midazolam and fentanyl combination in terms of analgesia, percentage of times having desired CPOT score: group A 81.57 \pm 6.48 vs. group B 84.64 \pm 7.47 , $p<0.05$. The requirement of open level fentanyl use was also higher in group A $p<0.05$. The incidence of delirium and the duration of mechanical ventilation was higher in group B $(\mathrm{p}<0.05)$. Conclusion: Dexmedetomidine is not as effective as midazolam \& fentanyl combination to achieve both sedation and analgesia in mechanically ventilated patients.

Key words: Dexmedetomidine, Sedation, Analgesia, Ventilated patient, GABA.

Copyright $(\mathcal{C} 2021$ The Author(s): This is an open-access article distributed under the terms of the Creative Commons Attribution 4.0 International License (CC BY-NC 4.0) which permits unrestricted use, distribution, and reproduction in any medium for non-commercial use provided the original author and source are credited.

\section{INTRODUCTION}

To reduce anxiety and pain, increase tolerance and improve outcomes of mechanically ventilated patients, sedation and analgesia are common practice. Over decades, $\gamma$-Aminobutyric acid (GABA) receptor agonist and opioids are used commonly for sedation and analgesia, respectively in intensive care unit (ICU). Preliminary evidence indicates that the $\alpha-2$ agonist dexmedetomidine may have distinct advantages to maintain both sedation and analgesia with minimal cardiovascular instability or respiratory depression, facilitate weaning from mechanical ventilation and decreases the duration of ICU stay. Pain, agitation, and delirium are very common sufferings of mechanically ventilated patients in the intensive care unit (ICU). Appropriate management of these three with adequate sedation and analgesia is a cornerstone of bedside care for most of the mechanically ventilated patients. It ensures patient comfort and safety [1]. Pain and anxiety 
contribute to the prominent sympathetic stress response that includes increased endogenous catecholamine activity, increased oxygen consumption, tachycardia, hypercoagulability, hyper metabolism, and immunosuppression [2]. These effects are increased by the ICU environment, mechanical ventilation, and various invasive interventions in critically ill patients. Unrelieved pain, anxiety and severe agitation lead to prolonged mechanical ventilation, increased incidence of delirium, length of ICU stay and the accidental removal of life-saving medical devices like endotracheal tubes and intravascular lines [3]. To ensure patient care, safety, comfort and to minimize the adverse outcomes due to pharmacotherapy, physicians must achieve the right balance of analgesic and sedative drug administration. The most commonly administered sedative for ICU patients worldwide is GABA agonists (including propofol and benzodiazepines such as midazolam), despite the well-known hazards associated with their prolonged use [4]. Benzodiazepines lead to tolerance, dependence, and arrhythmia, cardiac and respiratory depression which increases the adverse outcome of the patients. On the other hand, propofol has no direct analgesic effect. It produces hypotension, bradycardia, propofol infusion syndrome, immunosuppression like serious side effects which contribute to patient's mortality [5]. Opioids remain the mainstay of choice for analgesic therapy in ICU patients [6]. But all opioids depress respiratory drive in a dosedependent manner, which is more prominent when they are combinedly used with benzodiazepines. Morphine is the most hydrophilic of the opioids, undergoes hepatic metabolism and is eliminated by the kidneys which may, therefore, accumulate in patients with renal dysfunction. For this reason, fentanyl is used more in patients with renal insufficiency [2]. Fentanyl is a fatsoluble drug which has a large volume of distribution with increased risks of accumulation and delayed recovery after prolonged administration. It also produces dependence and withdrawal effect of the patients [7]. Dexmedetomidine is a potent and highly selective $\alpha 2$-adrenoceptor agonist with an extensive range of pharmacological properties and a unique mechanism of action [7]. Besides the various benefits during the postoperative period, it provides sedation and anxiolysis and attenuation of the stress response with no significant respiratory depression or delirium [8]. Several studies showed its effect of organ protection, and cognitive preservation in critically ill patients [9]. It was not recommended for long-term sedation previously. The randomized, double-blind, multi-Centre MIDEX and PRODEX trials indicated that longer-term sedation with dexmedetomidine was non-inferior to midazolam and propofol, which means both short- and longer-term use of this agent are suitable in ICU patients [10]. Moreover, compared with midazolam or propofol, patients receiving dexmedetomidine were also more comfortable to rouse, and more cooperative to communicate. Direct stimulation of $\alpha-2$ adrenoceptors in the spinal cord by dexmedetomidine causes the analgesic effect. It has a short onset of sedation, an elimination half-life of nearly two hours, negligible drug-drug interaction potential, and complete elimination within 10 hours after infusion termination, which favours highlighting its clinical use [11]. Since inception, midazolam and fentanyl have shown its efficacy for sedation and analgesia in several studies. However, ICU patients have unpredictable pharmacokinetics and pharmacodynamics due to hemodynamic instability, drug interactions, altered protein binding, and impaired organ function [12]. These factor increases the risk of complications when midazolam and fentanyl are co-administration. To develop the best treatment strategy for analgesia and sedation, the specific medical condition necessitating treatment must be recognized and continually reevaluated $[2,8]$. However, to date, there is a very minimal study regarding dexmedetomidine use as a single agent for both sedation and analgesia. Therefore, this study was designed to assess the effectiveness of dexmedetomidine than the use of midazolam and fentanyl combination for sedation and analgesia in mechanically ventilated patients.

\section{Methodology \& Materials}

This observational comparative study was conducted in the ICU of Dhaka Medical College Hospital (DMCH) at the Department of Anaesthesia, Analgesia \& Intensive Care Medicine during the period from March 2017 to June 2019. Total 146 mechanically ventilated patients were included in the study according to the selection criteria. Richmond agitation-sedation scale (RASS) and the Critical-care pain observation tool (CPOT) were used for assessment of sedation and analgesia respectively in this study. Ethical issues were ensured and written informed consent was taken before data collection from the legal guardian. After fulfilling the inclusion and exclusion criteria, the nature and benefit of the study were explained to the patient's guardian in details. Purposive sampling was done. All study subjects were blinded to their treatment. At first, the patients were divided into two groups and denoted as group A, and group B. Patients receiving Dexmedetomidine were denoted as in group a, and those receiving midazolam and fentanyl combined were included into group B. Randomization by fix card lottery method. According to the inclusion criteria of this study patient's age $\geq 18$ years, patients intubated and mechanically ventilated before the study and patients with anticipated ventilation, sedation duration of at least three more days were included. On the other hand, according to the exclusion criteria of this study, trauma or burn patients, patients are on dialysis, patient having epidural or spinal analgesia, patients with uncontrolled seizures, severe dementia, severe liver disease (Child-Pugh class C), pregnant or lactating mother, patients with cardiac abnormalities with left ventricular ejection fraction less than $30 \%$, heart rate less than 50/min, second- or third-degree heart block, and patients receiving continuous infusions of 2 
vasopressors to maintain blood pressure were excluded from the study. The group-A received Dexmedetomidine, and group-B receive Midazolam and Fentanyl combination after intubation. Sedatives and analgesic used before study enrollment were discontinued before the initiation of study drug, and patients were assessed initially by Richmond Agitation, and Sedation Scale (RASS) and Critical-care pain observation tool (CPOT). Loading doses for group-A was 0.4 to $1 \mu \mathrm{g} / \mathrm{kg}$ dexmedetomidine over 10 minutes and for group-B was 0.01 to $0.05 \mathrm{mg} / \mathrm{kg}$ midazolam was given according to severity of baseline RASS score. The starting maintenance infusion dose was $0.7 \mu \mathrm{g} / \mathrm{kg}$ per hour for dexmedetomidine and $0.06 \mathrm{mg} / \mathrm{kg}$ per hour for midazolam, corresponding to the midpoint of the allowable infusion dose range 0.4 to $1 \mu \mathrm{g} / \mathrm{kg} / \mathrm{hr}$ and 0.02 to $0.1 \mathrm{mg} / \mathrm{kg} / \mathrm{hr}$ respectively. For group B, fentanyl bolus doses 0.5 to $1.0 \mu \mathrm{g} / \mathrm{kg}$ according to severity of baseline CPOT score and maintenance infusion dose 0.7 to $1 \mu \mathrm{g} / \mathrm{kg} / \mathrm{hr}$ were administered. Assessment of the patients was done three times daily up to extubation or up to 3 days following administration of study drugs. Dosing of study drug was also adjusted, based on routine assessment findings and assessment of other treating physicians by the same tools. In case of inadequate sedation, both groups of patients could receive open-label midazolam bolus doses of 0.01 to $0.05 \mathrm{mg} / \mathrm{kg}$ according to severity of RASS scores at 10to 15-minute intervals until adequate sedation (RASS range, -2 to +1 ) were achieved with a maximum dose of $4 \mathrm{mg}$ in 8 hours. In the case of over sedation (RASS range, -3 to -5 ), the infusion dose was decreased until patients returned to the acceptable sedation range. In the case of inadequate analgesia, both groups of patients could receive open- label bolus doses of $0.5-1.0 \mu \mathrm{g} / \mathrm{kg}$ fentanyl according to severity of CPOT scores. A daily awaking trial was performed during which patients within the RASS range -2 to +1 . They were asked to perform 4 tasks (open eyes to voice command, track investigator with eyes, squeeze a hand, and stick out tongue). Patients were considered awake with successful completion of the assessment when they could perform 3 of 4 tasks. Delirium was assessed daily during the arousal assessment with patients in the RASS range of $(-2$ to +1$)$ using the Confusion Assessment Method for the ICU (CAM-ICU). Safety was assessed by monitoring vital signs and physical examination findings. In this study, no other sedatives or analgesics and no oral form, as well as patch, were allowed during the study period. Intravenous haloperidol was considered for the treatment of agitation or delirium in increments of 1 to $5 \mathrm{mg}$, repeated every 10 to 20 minutes as needed, following consultation of the supervisor. Study drug infusion was stopped at the time of extubation in both groups if the investigator felt it would be the best interest of the patient. In all cases, two separate data collection sheet was used for both groups of patients, respectively, with maintaining confidentiality. Highest level of confidentiality and ethical standard were maintained during storage and analysis of the data. In this study, age in years, sex, height and ideal body weight were the demographic variables. Richmond agitation sedation scale (RASS) and Critical-care pain observation tool (CPOT) were used as the Sedation and analgesia scoring. Chief complains, clinical examination, cause of ICU transfer and duration of mechanical ventilation were considered as the major information about mechanical ventilation. Percentage of time within the target sedation (RASS score -2 to +1$)$ and analgesic $($ CPOT $<3$ ) range during the treatment period were considered as the primary outcome variables of this study. On the other hand, prevalence of delirium and the requirement of use of fentanyl and open-label midazolam Duration of mechanical ventilation were the secondary outcome variables here. After collection of all the required data, these were checked, verified for consistency and then tabulated into the computer using the Package for Social Sciences (SPSS Inc., Chicago, IL, and version 22.0 for Windows. For normally distributed data, means were compared using Student's t-test for two groups. Qualitative or categorical variables were described as frequencies and proportions. Proportions were compared using the Chi- square test whichever applicable. All statistical tests were two-sided and performed at a significance level of $\mathrm{p}=<0.05$.

\section{RESULT}

Total 150 patients admitted in Anaesthesia, Analgesia, Palliative \& Intensive Care Medicine unit, Dhaka Medical College Hospital and fulfilled inclusion and exclusion criteria were included in the study in the first stage. They were subdivided into two groups. Eighty-two patients were in Group A and received dexmedetomidine. Rests 68 were in group B and received midazolam and fentanyl combination. However, within three days, a total of 4 patients (3 in group A and 1 in group B) died. So, finally, 146 cases were enrolled in the primary analysis. Among them, 79 were in group A, and 67 were in group B. The main aim of the study was to explore the effectiveness of dexmedetomidine for both sedation and analgesia in mechanically ventilated patients. Values were expressed as Mean $\pm \mathrm{SD}$ and within parenthesis percentage (\%) over the column in total. Chi-squared Test ( $\square 2)$ was performed to compare between two groups. Student ttest was performed to compare the mean age of both groups. There had no significant difference in the age height weight and gender distribution as $\mathrm{p}>0.05$. In analyzing the causes of referral in ICU we did not find statistically significant difference between two groups $(\mathrm{p}=0.40)$. On the other hand, in analyzing the systemrelated condition of referral in ICU among 146 study cases we observed, maximum $39(26.7 \%)$ and 30 $(20.5 \%)$ were referred to ICU for respiratory-related condition and infectious disease condition respectively. Between the groups, there had no statistically significant difference in causes $(\mathrm{p}=0.927)$. In analyzing patients completing all daily arousal assessment and incidence of delirium in participants we observed, the 
Nasir Uddin Ahmed et al; Sch J App Med Sci, Jan, 2021; 9(1): 149-155

completion rate was statistically significantly higher in group A than group B $(\mathrm{p}=0.036)$. Incidence of delirium was statistically significantly $(\mathrm{p}<0.001)$ lower in group A than group B. In analyzing the requirement of openlabel fentanyl and midazolam among participants we observed, between the groups, there had no significant difference regarding open-label midazolam use $(\mathrm{p}=$ 0.431). But the requirement of using fentanyl was statistically significantly lower in group $B(p=0.002)$. In analyzing the outcome of patients heart rate and blood pressure among the participants we observed, Group A patients were significantly more prone to develop hypotension and bradycardia $(\mathrm{p}<0.05)$. However, there had no statistically significant difference in developing hypertension and tachycardia between the groups
( $p>0.05)$. According to the patient data the duration of mechanical ventilation was found statistically significantly more in group B than group A $(p=0.002)$. Group $\mathrm{A}$, there were moderate negative correlation between duration of mechanical ventilation with the percentage of times having desired RASS score, It was highly significant, as $\mathrm{p}<0.001$. Besides, in group B there had week negative correlation between duration of mechanical ventilation with the percentage of times having desired RASS score and it was not significant, as $\mathrm{p}=0.102$. Among group $\mathrm{A}$ and group $\mathrm{B}$, there were negligible negative correlation between duration of mechanical ventilation with the percentage of times having desired CPOT score, not significant, as $\mathrm{p}=.731$ and $\mathrm{p}=0.181$ respectively.

Table-I: Causes of referral in ICU $(n=146)$

\begin{tabular}{|l|l|l|l|l|l|l|l|}
\hline \multirow{2}{*}{ Cause } & \multicolumn{2}{|c|}{ Group A $(\mathbf{n}=\mathbf{7 9})$} & \multicolumn{2}{|c|}{ Group B $(\mathbf{n}=67)$} & \multicolumn{2}{|c|}{ Total $(\mathbf{n}=\mathbf{1 4 6})$} & \multirow{2}{*}{ P-value } \\
\cline { 2 - 7 } & $\mathrm{n}$ & $\%$ & $\mathrm{n}$ & $\%$ & $\mathrm{n}$ & $\%$ & \\
\hline Medical & 41 & 51.90 & 37 & 55.22 & 78 & 53.42 & \multirow{2}{*}{0.40} \\
\hline Surgical & 38 & 48.10 & 30 & 44.78 & 68 & 46.58 & \\
\hline
\end{tabular}

Table-II: System-related condition of referral in ICU $(n=146)$

\begin{tabular}{|l|l|l|l|l|}
\hline Cause & Group A & Group B & Total & \multirow{2}{*}{ P-value } \\
\cline { 2 - 4 } & $\mathrm{n}(\%)$ & $\mathrm{n}(\%)$ & $\mathrm{n}(\%)$ & \\
\hline Cardiovascular disease & $11(13.9)$ & $9(13.4)$ & $20(13.7)$ & \multirow{2}{*}{$\mathbf{0 . 9 2 7}$} \\
\hline Respiratory disease & $22(27.8)$ & $17(25.4)$ & $39(26.7)$ & \\
\hline Renal disease & $7(8.9)$ & $4(6)$ & $11(7.5)$ & \\
\hline Head Injury & $12(15.2)$ & $11(16.4)$ & $23(15.8)$ & \\
\hline Infectious disease & $16(20.3)$ & $14(20.9)$ & $30(20.5)$ & \\
\hline Obs. \& Gyne. related condition & $10(12.7)$ & $9(13.4)$ & $19(13)$ & \\
\hline Malignancy & $1(1.3)$ & $3(4.5)$ & $4(2.7)$ & \\
\hline
\end{tabular}

Table-III: Patients completing all daily arousal assessment and incidence of delirium in participants $(n=146)$

\begin{tabular}{|l|l|l|l|l|}
\hline \multirow{2}{*}{ Component } & Group A & Group B & Total & \multirow{2}{*}{ P-value } \\
\cline { 2 - 4 } & $\mathbf{n}(\boldsymbol{\%})$ & $\mathbf{n}(\boldsymbol{\%})$ & $\mathbf{n}(\boldsymbol{\%})$ & \\
\hline Completing all daily arousal assessment & $71(89.9)$ & $52(77.6)$ & $123(84.2)$ & 0.036 \\
\hline Delirium & $16(20.03)$ & $40(59.7)$ & $56(38.4)$ & $<0.001$ \\
\hline
\end{tabular}

Table-IV: The requirement of open-label fentanyl and midazolam among participants (n=146)

\begin{tabular}{|l|l|l|l|r|}
\hline \multirow{2}{*}{ Requirement } & Group A & Group B & Total & \multirow{2}{*}{ P-value } \\
\cline { 2 - 4 } & n (\%) & n (\%) & n (\%) & \\
\hline Open-label midazolam & $18(22.80)$ & $17(25.40)$ & $35(24)$ & 0.431 \\
\hline Open-label Fentanyl & $21(26.60)$ & $05(7.5)$ & $26(17.8)$ & 0.002 \\
\hline
\end{tabular}

Table-V: Outcome of patients heart rate and blood pressure $(n=146)$

\begin{tabular}{|l|l|l|l|l|}
\hline \multirow{2}{*}{ Status } & Group A & Group B & Total & \multirow{2}{*}{ p-value } \\
\cline { 2 - 4 } & $\mathbf{n}(\boldsymbol{\%})$ & $\mathbf{n}(\boldsymbol{\%})$ & $\mathbf{n}(\boldsymbol{\%})$ & \\
\hline Hypotension & $38(48.1))$ & $14(20.9)$ & $52(35.6)$ & 0.001 \\
\hline Hypertension & $15(19)$ & $11(16.40)$ & $26(17.80)$ & 0.427 \\
\hline Bradycardia & $32(40.5)$ & $11(16.4)$ & $43(29.5)$ & 0.001 \\
\hline Tachycardia & $13(16.5)$ & $12(17.90)$ & $25(17.10)$ & 0.494 \\
\hline
\end{tabular}

Table-VI: Comparison of groups with the duration of mechanical ventilation of the patients $(n=146)$

\begin{tabular}{|l|l|l|l|}
\hline Variable & Group A & Group B & P-value \\
\hline Duration of mechanical & $4.1519( \pm .83)$ & $4.6418( \pm 1.03)$ & 0.002 \\
\hline
\end{tabular}


Nasir Uddin Ahmed et al; Sch J App Med Sci, Jan, 2021; 9(1): 149-155

Table-VII: Correlation between duration of mechanical ventilation with the percentage of times having desired RASS score $(n=146)$

\begin{tabular}{|l|l|l|l|l|l|l|}
\hline \multirow{2}{*}{ Parameter } & \multicolumn{4}{|l|}{ Group A } & \multicolumn{3}{l|}{ Group B } \\
\cline { 2 - 7 } & R & R2 & P value & R & R2 & P value \\
\hline Duration of mechanical ventilation & -0.639 & 0.41 & $<0.001$ & -0.202 & 0.041 & 0.102 \\
\hline
\end{tabular}

Table-VIII: Correlation between duration of mechanical ventilation with the percentage of times having desired CPOT score $(n=146)$

\begin{tabular}{|l|l|l|l|l|l|l|}
\hline \multirow{2}{*}{ Parameter } & \multicolumn{2}{|l|}{ Group A } & \multicolumn{3}{l|}{ Group B } \\
\cline { 2 - 7 } & R & R2 & P value & R & R2 & P value \\
\hline Duration of mechanical ventilation & -0.039 & 0.002 & 0.731 & -0.165 & 0.027 & 0.181 \\
\hline
\end{tabular}

\section{DISCUSSION}

The aim of this study was to assess the effectiveness of dexmedetomidine to the combined use of midazolam and fentanyl for sedation and analgesia in mechanically ventilated patients. The study was conducted to assess the effectiveness of dexmedetomidine for sedation and analgesia in mechanically ventilated patients. According to the study, there had no significant difference in the age distribution between group A and group B $(\mathrm{p}=0.589)$. Maximum 52(35.6\%) study cases were in 31-40 years age group with mean $46.11 \pm 13.37$ years. This finding was consistent with the finding of Alam and Haque [13]. The lower mean age may be due to geographical variations, racial, ethnic differences, and genetic causes. Among the cases, $53.4 \%$ were referred to ICU for medical causes and rests were referred for surgical causes. This reflects the need for mechanical ventilation in general ICU like DMCH almost equal in medical and surgical cases. This finding was similar to the finding of Ahmed et al. [14]. This study revealed respiratory disease and infections contributes to a large number of ICU referral for mechanical ventilation. This finding was also similar to the finding of Hossain and Maruf [15]. It also supports a higher incident of sepsis in $\mathrm{DMCH}$, ICU and major concern patient management. The desired sedation level by RASS score was more percentage of time in group A than group B $(\mathrm{p}<0.001)$. In group $\mathrm{A}$, the desired RASS score was present in $85.23( \pm 7.54) \%$ of the time, and group B desired RASS score was present in $79.19( \pm 7.84) \%$ of the time. This finding was similar to the study of Jakob et al. [10]. Dexmedetomidine binds only selective $\alpha 2$ receptors rather than GABA receptor which provide light sedation, sympatholytic action, blunting of the stress, less respiratory depression and established the more natural sleep-like state. On the other hand, midazolam produces deep sedation and severe impaired cognitive function which produce RASS range below 2. This may be the explanation of adequate sedation outcome of dexmedetomidine in this study. This finding also matches with the finding of Riker et al. [16]. In the case of analgesia, between the groups, there had a statistically significant difference in the CPOT score $\mathrm{p}<0.05$. Adequate analgesia maintained more in group $B$ patients. On the other hand, the study conducted by Fahmy et al., [17] found a significant positive result in the first 24 hours between two groups regarding the CPOT score. Fentanyl infusion was not used their study in the midazolam group. They gave only open level fentanyl in both groups. For that equal amount of fentanyl in both groups facilitated their significant result. In this study infusion of fentanyl and also open level fentanyl were used in the midazolam group which is the cause of more analgesic effect of group B. Daily awakening assessment rate was significantly higher in group $\mathrm{A}$ than group $\mathrm{B}(\mathrm{p}=0.036)$. In group A 71 $(89.9 \%)$ patients completed all daily arousal assessment and in group B $52(77.6 \%)$ patients completed all daily arousal assessment. Riker et al., [16] also found similar results in their study. Dexmedetomidine produces light sedation with a minimal cognitive impairment which helps the patient to arouse quickly. This is the reflection of the daily arousal assessment results of this study. Incidence of delirium is statistically significantly more in group B than group A. In group B 40 (59.7\%) patients had developed delirium, and in the group, an only $16(20.3 \%)$ patients had developed delirium. Studies conducted by Peng et al. [18] also shown that prevalence of delirium was significantly $(p<0.001)$ lower in the dexmedetomidine group than midazolam group. Midazolam has a high affinity of GABA receptors. The activation of these receptors alters deliriogenic neurotransmitters which impair the quality of sleep and leads delirium [19]. This described the higher incidence of delirium in the study. The requirement of using open-label fentanyl was statistically significantly lower in group $B(p=0.002)$. During the study, group B patients received fentanyl routinely. So it may be the possible reason why group a patients need more open level fentanyl than group B patients. However, between the groups, there had no statistically significant difference found regarding using of open-label midazolam for gaining adequate sedation (0.431). This finding is similar to the finding of Jakob and co-researchers et al.,[10]. Group A patients were significantly more prone to develop hypotension and bradycardia $(p<0.05)$ than group B patients [20] also noticed a significant fall of blood pressure in the dexmedetomidine group than midazolam group in their study. Dexmedetomidine has sympatholytic action which helps normalized increased level of stress produced by mechanical ventilation. This is maybe one of the reasons for hypotension and bradycardia. Duration of mechanical ventilation was significantly 
more noticed in group B patients than group A patients $(p=0.002)$. This finding is similar to the study of Riker and his collogues [16]. Dexmedetomidine produces only mild cognitive impairment, allowing easy communication. It does not affect respiratory drive for that no interferes with weaning from mechanical ventilation. For that there was significant negative correlation of duration of mechanical ventilation with the percentage of times having desired RASS score patient receiving dexmedetomidine. This is also beneficial for health care cost, increases ICU bed availability and decreases the adverse outcome of the patient [17]. So the study reveals that despite some adverse effects like hypotension and bradycardia dexmedetomidine group had a better outcome than the midazolam-fentanyl group. In the dexmedetomidine group, the incidence of delirium and, duration of mechanical ventilation was significantly low. Desired RASS score and arousal status of the patients were also significantly high in the dexmedetomidine group.

\section{Limitations of the study}

This was a single centered study with a small sized sample. So the findings of this study may not reflect the exact scenario of the whole country. Besides these, short term follow-up procedure was also a limitation of this study.

\section{Conclusion and Recommendations}

In this study, it was observed that dexmedetomidine is not adequately effective than the use of midazolam and fentanyl combination to achieve both sedation and analgesia in mechanically ventilated patients. The findings of this study may be helpful in similar further studies. But for getting more specific findings we would like to recommend for conducting more studies regarding the same issue in several places.

\section{REFERENCES}

1. Ge XY, Huang Y, Yan XD, Cheng W. Dexmedetomidine: therapeutic efficacy in adult patients of the intensive care unit. International journal of clinical and experimental medicine. 2017 Jan 1;10(2):2016-25.

2. Jacobi J. Task Force of the American College of Critical Care Medicine (ACCM) of the Society of Critical Care Medicine (SCCM), American Society of Health-System Pharmacists (ASHP), American College of Chest Physicians. Clinical practice guidelines or the sustained use of sedatives and analgesics in the critically ill adult. Crit Care Med. 2002;30:119-41.

3. Brush DR, Kress JP. Sedation and analgesia for the mechanically ventilated patient. Clinics in chest medicine. 2009 Mar 1;30(1):131-41.

4. Ouimet S, Kavanagh BP, Gottfried SB, Skrobik Y. Incidence, risk factors and consequences of ICU delirium. Intensive care medicine. 2007 Jan 1;33(1):66-73.
5. Bennett SN, McNeil MM, Bland LA, Arduino MJ, Villarino ME, Perrotta DM, Burwen DR, Welbel SF, Pegues DA, Stroud L, Zeitz PS. Postoperative infections traced to contamination of an intravenous anesthetic, propofol. New England Journal of Medicine. 1995 Jul 20;333(3):147-54.

6. Soliman HM, Mélot C, Vincent JL. Sedative and analgesic practice in the intensive care unit: the results of a European survey. British journal of anaesthesia. 2001 Aug 1;87(2):186-92.

7. Panzer O, Moitra V, Sladen RN. Pharmacology of sedative-analgesic agents: dexmedetomidine, remifentanil, ketamine, volatile anesthetics, and the role of peripheral mu antagonists. Anesthesiology clinics. 2011 Dec 1;29(4):587-605.

8. Adams R, Brown GT, Davidson M, Fisher E, Mathisen J, Thomson G, Webster NR. Efficacy of dexmedetomidine compared with midazolam for sedation in adult intensive care patients: a systematic review. British journal of anaesthesia. 2013 Nov 1;111(5):703-10.

9. Ma D, Hossain M, Rajakumaraswamy N, Arshad M, Sanders RD, Franks NP, Maze M. Dexmedetomidine produces its neuroprotective effect via the $\alpha 2 \mathrm{~A}$-adrenoceptor subtype. European journal of pharmacology. 2004 Oct 11;502(1-2):8797.

10. Jakob SM, Ruokonen E, Grounds RM, Sarapohja T, Garratt C, Pocock SJ, Bratty JR, Takala J, Dexmedetomidine for Long-Term Sedation Investigators. Dexmedetomidine vs midazolam or propofol for sedation during prolonged mechanical ventilation: two randomized controlled trials. Jama. 2012 Mar 21;307(11):1151-60.

11. Piao G, Wu J. Systematic assessment of dexmedetomidine as an anesthetic agent: a metaanalysis of randomized controlled trials. Archives of medical science: AMS. 2014 Feb 24;10(1):19.

12. Liu ZX, Xu FY, Liang X, Zhou M, Wu L, Wu JR, Xia JH, Zou Z. Efficacy of dexmedetomidine on postoperative shivering: a meta-analysis of clinical trials. Canadian Journal of Anesthesia/Journal canadien d'anesthésie. 2015 Jul;62(7):816-29.

13. Alam MR, Haque M, Haque M. An Appraisal of mortality in intensive care unit of a Level III Military hospital of bangladesh. Indian Journal of Critical Care Medicine: Peer-reviewed, Official Publication of Indian Society of Critical Care Medicine. 2017 Sep;21(9):594.

14. Ahmed S, Yusuf OF, Alam AS, Awal A. Profile of Intensive Care Unit admission and Outcomes of Medical and Surgical patients at A Tertiary Government Hospital: A 5 Year Trend Analysis. Bangladesh Journal of Medicine. 2018 Aug 18;29(2):59-62.

15. Hossain MD, Al Maruf A. An Analysis of 1255 ICU Patients at a Tertiary Military Hospital. Bangladesh Critical Care Journal. 2013 Mar $31 ; 1(1): 40-4$. 
16. Riker RR, Shehabi Y, Bokesch PM, Ceraso D, Wisemandle W, Koura F, Whitten P, Margolis BD, Byrne DW, Ely EW, Rocha MG. Dexmedetomidine vs midazolam for sedation of critically ill patients: a randomized trial. Jama. 2009 Feb 4;301(5):489-99.

17. Abdellatif AE, Fahmy NM, Abdel Hamid HS, Rabie AH, Salib HM. Dexmedetomedine versus Standard Sedatives in Weaning from Mechanical Ventilation. The Egyptian Journal of Hospital Medicine. 2018 Jul 1;72(4):4292-9.
18. Peng W, Shimin S, Hongli W, Yanli Z, Ying Z. Delirium risk of dexmedetomidine and midazolam in patients treated with postoperative mechanical ventilation: a meta-analysis. Open Medicine. 2017 Aug 10;12(1):252-6.

19. Pandharipande PP, Pun BT, Herr DL, Maze M, Girard TD, Miller RR, Shintani AK, Thompson JL, Jackson JC, Deppen SA, Stiles RA. Effect of sedation with dexmedetomidine vs lorazepam on acute brain dysfunction in mechanically ventilated patients: the MENDS randomized controlled trial. Jama. 2007 Dec 12;298(22):2644-53. 